\title{
MAGNETIC MEASUREMENTS AND SIMULATIONS FOR A 4-MAGNET DIPOLE CHICANE FOR THE INTERNATIONAL LINEAR COLLIDER
}

\author{
Ray Arnold, Andrew Fisher, Carsten Hast, Zen Szalata, Mike Woods, SLAC \\ Heinz Juergen Schreiber, Michele Viti, DESY \\ Viktor Duginov, Sergey Kostromin, Nikolay Morozov, JINR
}

\section{Abstract}

T-474 at SLAC is a prototype BPM-based energy spectrometer for the ILC. We describe magnetic measurements and simulations for the 4-magnet chicane used in T-474.

\section{INTRODUCTION}

The ILC physics program requires better than 100 part-per-million (ppm) accuracy for energy measurements, which necessitates better than $50 \mathrm{ppm}$ accuracy for magnetic field integral measurements. A 4dipole chicane is used in T-474 with mid-chicane dispersion of $5-\mathrm{mm}$ and magnetic fields of $\sim 1 \mathrm{kGauss}$; similar to the current ILC parameters.

Stability, reproducibility and consistency of magnetic measurements, including magnetic field maps for the T474 dipole magnets, are presented using a moving wire, rotating coil, NMR probe, Hall probe and low-field fluxgate magnetometer.

Measurements from SLAC's Magnet Test Lab facility as well as in situ measurements in End Station A (ESA) are presented, including measurements of residual magnetic fields in the T-474 chicane between the chicane magnets. Results are provided for an operational mode with a 1-hour calibration cycle, where the chicane magnets are operated in both polarities and at near-zero field.

\section{SLAC T-474 ENERGY SPECTROMETER}

The concept of the energy measurement spectrometer is based on the determination of the bending angle of a charged particle through a dipole magnetic field. The particle energy is proportional to the integrated field and inversely proportional to the bend angle $\theta$ :

$$
E=\frac{c e}{\theta} \int B \partial l \text {, where } c \text { is the speed of light and } e \text { is }
$$

the charge of the particle.
The SLAC Linac delivers to ESA a $28.5 \mathrm{GeV}$ test beam with similar parameters as the ILC for bunch charge, bunch length and energy spread [1]. ESA beam tests run parasitically with PEP-II with single damped bunches at $10 \mathrm{~Hz}$, bunch charge of $1.6 \cdot 10^{10}$ electrons, rms bunch length $\sim 500$ microns and rms bunch energy spread $\sim 0.2 \%$.

The T-474 energy spectrometer is located in the ESA beamline (see fig. 1) and consists of four 10D37 dipole bending magnets and a number of BPM doublets and triplets. The magnets are 37" in length with 10 "-wide pole faces. The vertical gap is 3". They are run in series from a single power supply.. One NMR probe is installed in each of the four magnets and a single Hall probe is installed in the first magnet 3B1. Two fluxgate magnetometers measure stray fields in the range -5 to +5 gauss in the last leg of the chicane between $3 \mathrm{~B} 3$ and 3B4.

\section{FIELD MEASUREMENTS}

Stability, reproducibility and consistency of the dipole magnetic fields were studied in SLAC's Magnetic Measurement Test Laboratory. Prior to each set of

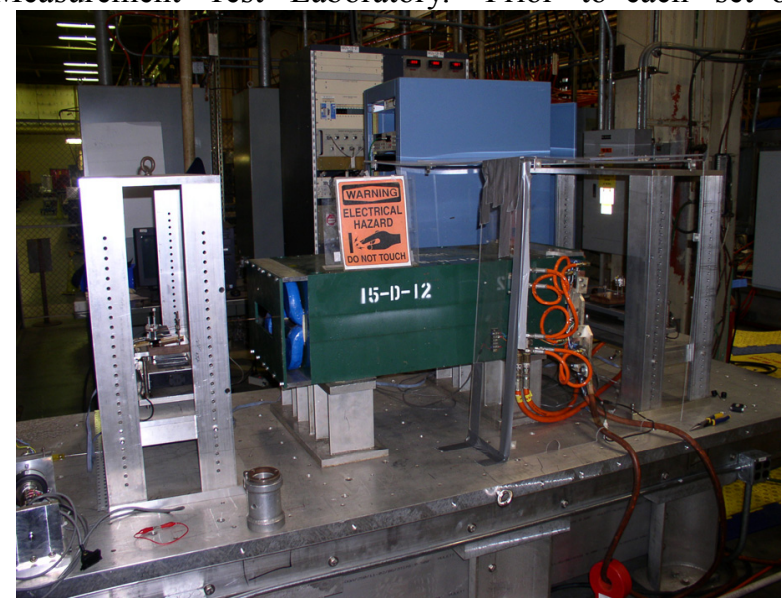

Figure 2: 10D37 dipole magnet on the test bench.

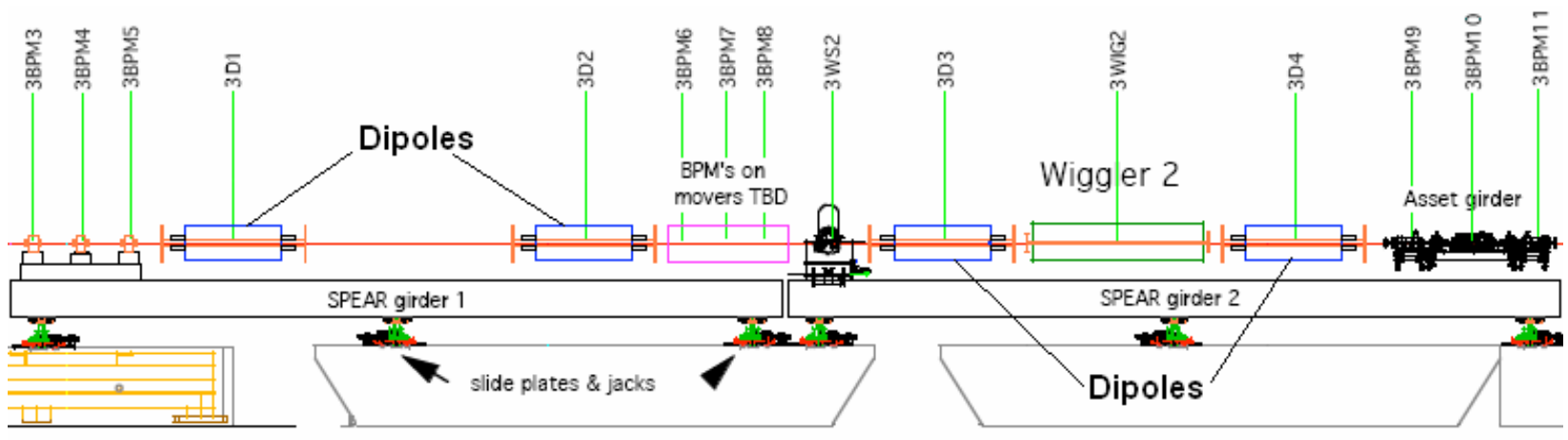

Figure 1: Scheme of the BPM-based energy spectrometer in ESA at SLAC. 
measurements, the magnets were run through a standardization procedure, defined to be operation at $+200 \mathrm{~A}$ for 5 minutes followed by 5 minutes operation at $200 \mathrm{~A}$ and then repeating this cycle 3 times. Standard operating currents for the magnets was $\pm 150 \mathrm{~A}$.

At the Test Lab, magnetic field maps of the vertical dipole field $B_{y}$ were taken using NMR and Hall probes up to 1-meter from the magnet center for each of the four magnets. The $\int B d l$ was measured using a 2 -meter long flip coil, which was calibrated against a moving wire system. Flip coil measurements were directly compared with NMR and Hall probe measurements during long stability runs and during simulated calibration runs where the magnet polarity was reversed. The temperature dependence of the magnetic field and $\int B d l$ was measured during a 24-hour stability run.

A scan of the power supply current from 140-150A for each magnet was used to obtain the relation from the NMR probe measurement to the $\int B d l$ determined from the flip coil. The relative stability of the difference $(\delta B d l$ $N M R)$ or $(B d l-H a l l)$ is observed to be $\sim 30 \mathrm{ppm}$ rms in a 6 -hour run at fixed current of $+150 \mathrm{~A}$ (see fig. 3).

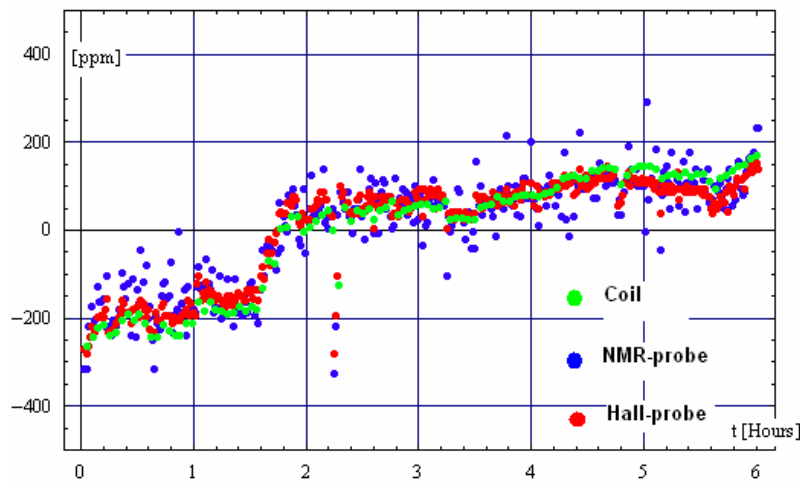

Figure 3: Stability of flux coil data, NMR and Hall measurements (in ppm, normalized to the mean values).

Figures 4 and 5 present results of the magnetic field mapping. It is necessary to take into account the magnetic field up to 1 meter from the dipole centre to determine $\int B d l$ to $50 \mathrm{ppm}$ accuracy.

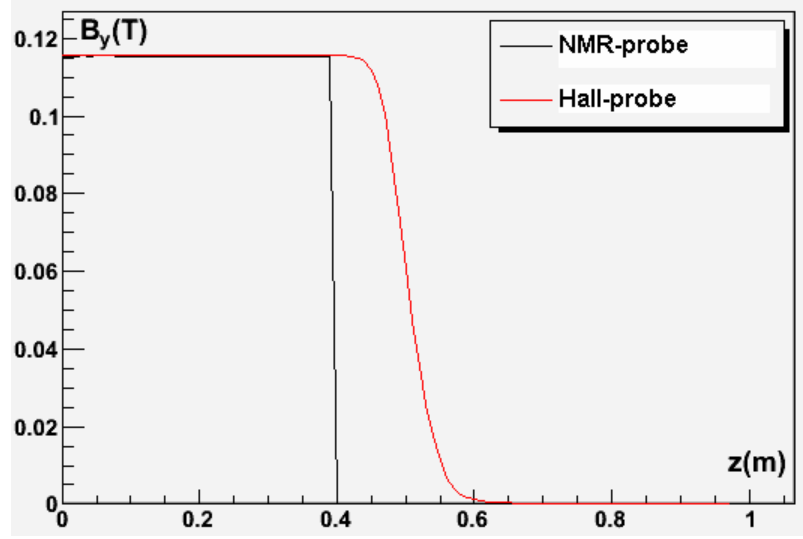

Figure 4: Vertical magnetic field map for one of the dipoles.
For ILC operation the energy spectrometer will be calibrated by reversing the chicane polarity and moving the mid-chicane BPMs by $\sim 10 \mathrm{~mm}$, tracked by interferometric measurements. In our tests, one calibration cycle is defined to be 20-minute operation at $+150 \mathrm{~A}$, followed by 20 -minute operation at $-150 \mathrm{~A}$. The relative stability of $\int B d l$ for the positive polarity runs for 6 calibration cycles is shown in Figure 6.

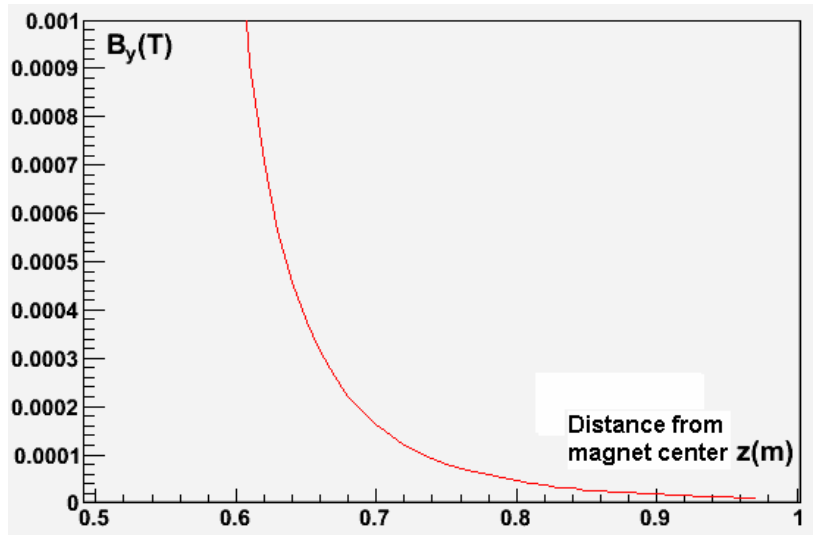

Figure 5: Magnetic field outside the magnet.

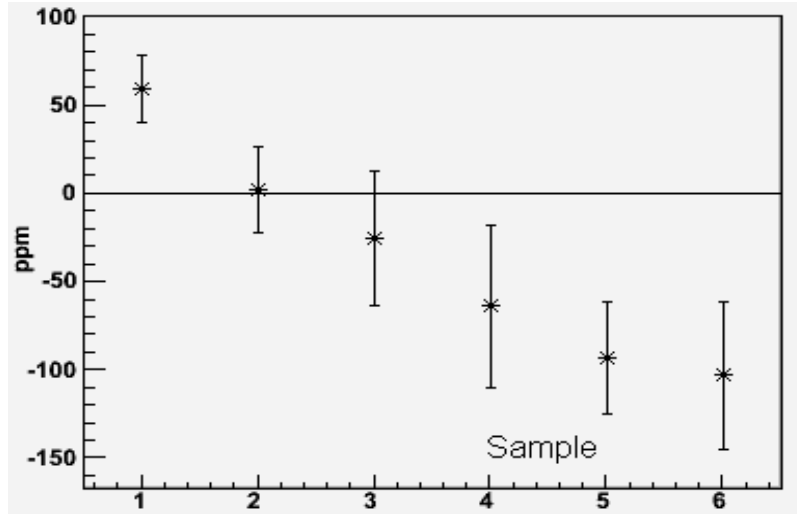

Figure 6: Relative stability for the $\int B d l$ during reproducibility test for one of the dipoles.

The agreement and stability of $\int B d l$-predicted from NMR probe data and actual $\int B d l$ measured by the flipcoil is plotted in Figure 7 during reproducibility run..

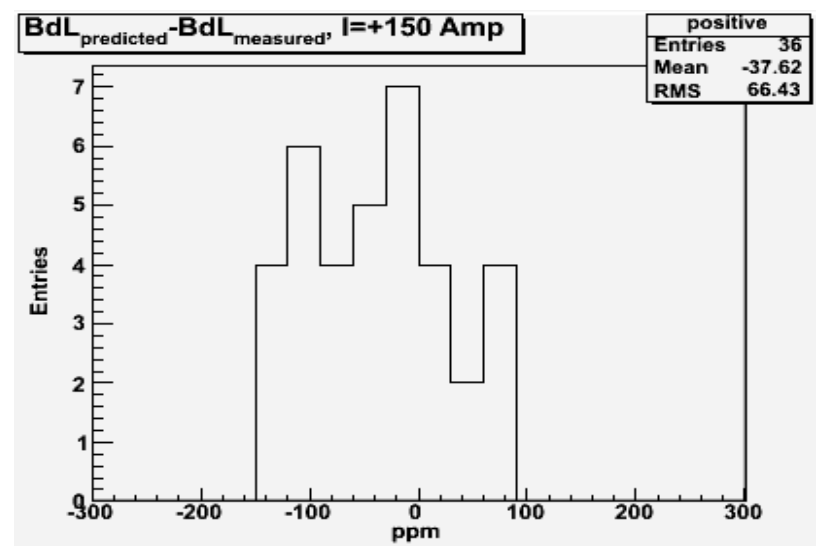

Figure 7: Agreement between predicted using control probe data $\int B d l$-value and the measured $\int B d l$-value. 
Other results obtained from the Test Lab include:

i) temperature dependence for $\int B d l$ is $57 \mathrm{ppm} / \mathrm{C}^{\circ}$, consistent with a simulation that gave $61 \mathrm{ppm} / \mathrm{C}^{\circ}$;

ii) measured and calculated magnetic fields for any of the dipoles is less than 7 Gauss for the field map data;

iii) $\mathrm{B}_{\mathrm{y}}$ is uniform to better than $100 \mathrm{ppm}$ for horizontal offsets $<15 \mathrm{~mm}$ from the magnet axis;

iv) difference between the $\int B d l$ measured by the flux coil and determined from the field map using NMR and Hall probes is about $20 \mathrm{ppm}$.

\section{ESA MEASUREMENTS}

A fluxgate magnetometer was used to measure the residual magnetic field in the T-474 chicane before installation of the dipoles for the March 2007 run. There are contributions from the earth's magnetic field of $\sim 0.5 \mathrm{G}$ and similar contributions from magnetized rebar in the support girders for the magnets.

The deflection angle $\theta$ of the beam was determined using rf BPMs shown in Figure 1 and an interferometer for tracking the $\sim 10 \mathrm{~mm}$ move of the mid-chicane BPM for the two chicane polarities. $\int B d l$ values were obtained using NMR probes and calibrations from the magnet test lab data.. Some results are presented below (see Figs. 8 and 9). BPM resolutions during these runs were $\sim 1 \mu \mathrm{m}[2]$.

Current estimate of the systematic error for monitoring $\int B d l$ in situ in ESA is $\sim 100 \mathrm{ppm}$. This includes uncertainties for the accuracy of the moving wire and flip coil technique, and for tracking the $\int B d l$ with the NMR and Hall probes, [3], [4].

The resolution of the energy measurement per bunch is determined by the BPM resolution of $\sim 1 \mu \mathrm{m}$, giving $\sim 230$ ppm (i. e. $6.6 \mathrm{MeV}$ in absolute scale).

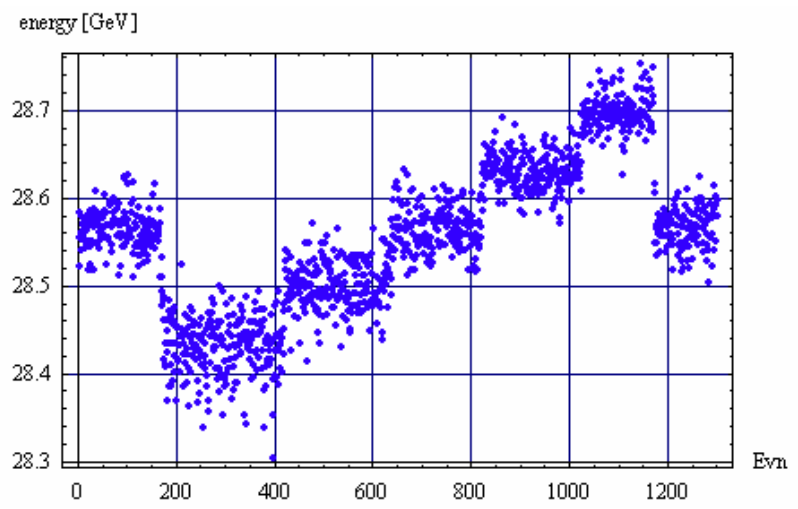

Figure 8: One of the energy scans during run-1699 at $-150 \mathrm{~A}$ - magnets current.

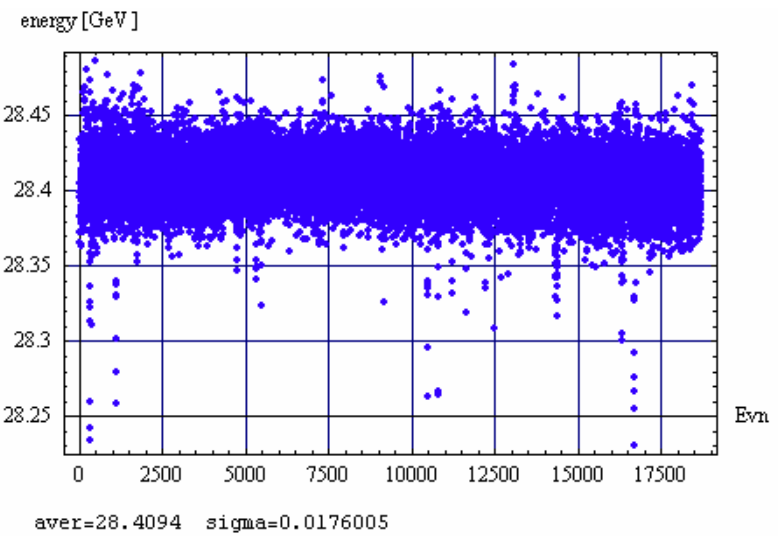

Figure 9: Energy measurement during Run-1955 (40 minutes stability run at $-150 \mathrm{~A}$ ).

\section{CONCLUSIONS}

Initial energy measurements with a prototype BPM spectrometer for the ILC were tested in T-474 at SLAC. Energy resolution per bunch is currently $\sim 230 \mathrm{ppm}$. Magnetic field maps and field integrals, as well as stability and reproducibility for these were investigated for each of the 4 dipoles used in a test lab. Standardization and energy calibration procedures were developed, which include reversing the chicane polarity for calibration. Accuracy of the magnetic field integral for each dipole with NMR monitoring is estimated to be $\sim 100 \mathrm{ppm}$ currently. A complete evaluation of systematic errors for in situ $\int B d l$ measurements and absolute energy scale for T474 is still underway.

T474 will continue with a run in summer 2007 and further runs in 2008. This experience will be used to complete the design for the ILC magnets, including their geometry, field measurement diagnostics and fabrication procedure.

\section{REFERENCES}

[1] M. Woods et al., "A test facility for the International Linear Collider at SLAC End Station A, for prototypes of beam delivery and IR components", SLAC-PUB-11180, EUROTEV-REPORT-2005-0081, PAC-2005-RPPP036, 2005. e-Print Archive: physics $/ 0505171$

[2] B. Maiheu et al., "A prototype energy spectrometer for the ILC at End Station A at SLAC", these proceedings.

[3] http://www.gmw.com/magnetic_measurements/ Grop3/documents/G3_MAN_DTM-151-S802010140.pdf

[4] http://www.gmw.com/magnetic measurements/ MetroLab/pdf/ML_MAN_PT2025-V2-R1Sep\%2003.pdf 